\title{
Automatic Image Annotation and Retrieval using Multi-Instance Multi-Label Learning
}

\author{
T. Sumathi, C. Lakshmi Devasena, R. Revathi, S. Priya and Dr.M. Hemalatha
}

\begin{abstract}
In this paper, we propose the MIML (MultiInstance Multi-Label learning) framework which is associated with multiple class labels for Image Annotation. Compared to traditional learning frameworks, the MIML framework is more convenient and natural for representing complicated objects which have multiple semantic meanings. To learn from MIML examples we have taken a survey on MIML Boost, MIMLSVM, D-MIMLSVM, InsDif and SubCod algorithms. MIML Boost and MIML SVM are based on a simple degeneration strategy. Experiments based on this algorithm shows that solving problems involving complicated objects with multiple semantic meanings in the MIML framework can lead to good performance. As the degeneration process may lose information, we have considered D-MimlSvm algorithm which tackles MIML problems directly in a regularization framework. InsDif and SubCod algorithms works by transforming single-instances into the MIML representation for learning, while SubCod works by transforming single-label examples into the MIML representation for learning. We have compared the results of all the algorithms and have identified that InsDifframework leads to good performance rates.
\end{abstract}

Keywords--- Machine Learning, Multi-Instance MultiLabel Learning, Multi-Label Learning, Multi-Instance Learning

\section{INTRODUCTION}

$\mathrm{I}_{\mathrm{b}}^{\mathrm{N}}$ $\mathrm{N}$ traditional supervised learning, an object is represented by an instance, i.e., a feature vector, and associated with a class label. Formally, let $\mathrm{X}$ denote the instance space (or feature space) and $Y$ the set of class labels. The task is to learn a function $\mathrm{f}: \mathrm{X} \rightarrow \mathrm{Y}$ from a given data set $\{(\mathrm{x} 1, \mathrm{y} 1),(\mathrm{x} 2, \mathrm{y} 2)$, $\ldots .,(\mathrm{xm}, \mathrm{ym})\}$, where $\mathrm{x}_{\mathrm{i}} \in \mathrm{X}$ is an instance and $\mathrm{y}_{\mathrm{i}} \in \mathrm{Y}$ is the known label of $\mathrm{X}_{\mathrm{i}}$. Although this formalization is prevailing and successful, there are many real-world problems which do not fit in this framework. Each object in this framework belongs to only one concept and therefore the corresponding instance is associated with a single class label. However, many real-world objects are complicated, which may belong to

T. Sumathi, Department of Software Systems, Karpagam University, Coimbatore-21.E-mail: tsumathijk@gmail.com

C. Lakshmi Devasena, Department of Software Systems, Karpagam University, Coimbatore-21.E-mail: devaradhe2007@gmail.com

$R$. Revathi, Department of Software Systems, Karpagam University, Coimbatore-21

S. Priya, Department of Software Systems, Karpagam University, Coimbatore-21

Dr.M. Hemalatha, Department of Software Systems, Karpagam University, Coimbatore-21.E-mail: hema.bioinf@gmail.com multiple concepts simultaneously. To choose the right semantic meaning for such objects for a specific scenario is the fundamental difficulty of many tasks. In contrast to starting from a large universe of all possible concepts involved in the task, it may be helpful to get the subset of concepts associated with the concerned object at first, and then make a choice in the small subset later. However, getting the subset of concepts, ie. assigning proper class labels to such objects is still a challenging task.

We notice that as an alternative to representing an object by a single instance, in many cases it is possible to represent a complicated object using a set of instances. For example, multiple patches can be extracted from an image where each patch is described by an instance, and thus the image can be represented by a set of instances. Using multiple instances to represent those complicated objects may be helpful because some inherent patterns which are closely related to some labels may become explicit and clearer. In this paper, we propose the MIML (Multi-Instance Multi-Label learning) framework, where an example is explained by multiple instances and correlated with multiple class labels.

Compared to traditional learning frameworks, the MIML framework is more convenient and natural for representing complicated objects. To exploit the advantages of the MIML representation, new learning algorithms are needed. We have compared the MIMLBoost algorithm and the MIMLSVM algorithm based on a simple degeneration strategy, and experiments show that solving problems involving complicated objects with multiple semantic meanings under the MIML framework can lead to good performance.

Considering that the degeneration process may lose information, we have compared the performance of $\mathrm{D}$ MIMLSVM (i.e., Direct MIMLSVM) algorithm which tackles MIML problems directly in a regularization framework.

In some practical tasks we do not have access to the real objects themselves such as the real images; instead, we are given observational data where each real object has already been represented by a single instance. Thus, in such cases we cannot capture more information from the real objects using the MIML representation. Even in this situation, however, MIML is still useful. We have also compared the performance of InsDif (i.e., INStance DIFferentiation) algorithm which transforms Single-instances into MIML examples for learning.

MIML can also be helpful for learning single-label objects. For which we have compared the SubCod (i.e., SUB-COncept Discovery) algorithm which works by discovering subconcepts of the target concept at first and then transforming 
the data into MIML examples for learning. This algorithm is able to achieve a better performance than learning the singlelabel examples directly in some tasks.

\section{RELATED WORK}

Much work has been devoted to the learning of multi-label examples under multi-label learning. Multi-label learning studies the problem where a real-world object described by one instance is associated with a number of class labels, which is different from multi-class learning or multi-task learning [3]. In multi-class learning each object is only associated with a single label; while in multi-task learning different tasks may involve different domains and different data sets. Actually, two-class and multi-class problems can both be transmit into multi-label problems by restricting that each instance has only one label. The generality of multi-label problems, however, inevitably makes it more difficult to address.

One famous approach for solving multi-label problems is Schapire and Singer's AdaBoost.MH [4], which is an extension of AdaBoost and is the core of a successful multilabel learning system BoosTexter [4]. This approach maintains a set of weights over both training examples and their labels in the training phase, where training examples and their corresponding labels that are hard (easy) to predict get incrementally higher (lower) weights. Later, De Comite et al. [5] used alternating decision trees [6] which are more powerful than decision stumps used in BoosTexter to handle multi-label data and thus obtained the AdtBoost.MH algorithm. Probabilistic generative models have been found useful in multi-label learning. McCallum [7] proposed a Bayesian approach for multi-label document classification, where a mixture probabilistic model (one mixture component per category) is assumed to generate each document and an EM algorithm is employed to learn the mixture weights and the word distributions in each mixture component.

Ueda and Saito [8] presented another generative approach, which assumes that the multi-label text has a mixture of characteristic words appearing in single-label text belonging to each of the multi-labels. It is noteworthy that the generative models used in [7] and [8] are both based on learning text frequencies in documents, and are thus specific to text applications.

Multi-instance learning techniques have already been applied to diverse applications including image categorization $[9,10]$, image retrieval $[11,12]$, text categorization $[13,14]$, web mining [15], spam detection [16], computer security [17], face detection [18, 19], computer-aided medical diagnosis [20], etc.

\section{THE MIML FRAMEWORK}

Let $\mathrm{X}$ denotes the instance space and $\mathrm{Y}$ the set of class labels. Then, formally, the MIML task is defined as:

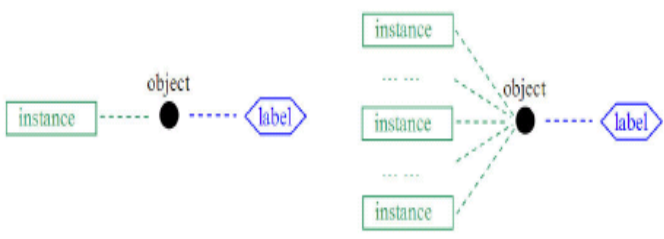

(a) Traditional supervised learning

(b) Multi-instance learning
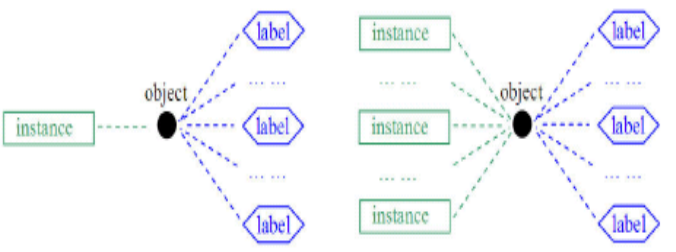

(c) Multi-label learning

(d) Multi-instance multi-label learning

Figure 1: Four Different Learning Framework

- MIML (Multi-Instance Multi-Label Learning): To learn a function $\mathrm{f}: 2 \mathrm{X} \rightarrow 2$ Yfrom a given data set $\{(\mathrm{X} 1, \mathrm{Y} 1),(\mathrm{X} 2, \mathrm{Y} 2), \ldots .,(\mathrm{Xm}, \mathrm{Ym})\}$, where $\mathrm{Xi} \subseteq \mathrm{X}$ is a set of instances $\{x i 1, x i 2, \ldots ., x i, n i\}, x i j \in X(j=$ $1,2, \ldots$, ni), and $\mathrm{Yi} \subseteq \mathrm{Y}$ is a set of labels $\{$ yi1, yi2, ...., yi,li $\},$ yik $\in \mathrm{Y}(\mathrm{k}=1,2, \ldots .$. li $)$. Here ni denotes the number of instances in $\mathrm{Xi}$ and li the number of labels in Yi. It is interesting to compare MIML with the existing frameworks of traditional supervised learning, multi-instance learning, and multi-label learning.

- Traditional Supervised Learning (Single-Instance Single-Label Learning): To learn a function $\mathrm{f}: \mathrm{X} \rightarrow \mathrm{Y}$ from a given data set $\{(\mathrm{x} 1, \mathrm{y} 1),(\mathrm{x} 2, \mathrm{y} 2) \ldots(\mathrm{Xm}, \mathrm{ym})\}$, where $\mathrm{xi} \in \mathrm{X}$ is an instance and yi $\in \mathrm{Y}$ is the known label of xi.

- Multi-Instance Learning (Multi-Instance Single-Label Learning): To learn a function $\mathrm{f}: 2 \mathrm{X} \rightarrow \mathrm{Y}$ from a given data set $\{(\mathrm{X} 1, \mathrm{y} 1),(\mathrm{X} 2, \mathrm{y} 2 \ldots(\mathrm{Xm}, \mathrm{ym})\}$, where $\mathrm{Xi} \subseteq \mathrm{X}$ is a set of instances $\{\mathrm{xi} 1, \mathrm{xi} 2 \ldots \mathrm{xi}, \mathrm{ni}\}, \mathrm{xij} \in$ $\mathrm{X}(\mathrm{j}=1,2, \ldots, \mathrm{ni})$, and $\mathrm{yi} \in \mathrm{Y}$ is the label of $\mathrm{Xi} .2$ Here $\mathrm{n}_{\mathrm{i}}$ denotes the number of instances in Xi.

- Multi-Label Learning (Single-Instance Multi-Label Learning): To learn a function $\mathrm{f}: \mathrm{X} \rightarrow 2 \mathrm{Y}$ from a given data set $\{(\mathrm{x} 1, \mathrm{Y} 1),(\mathrm{x} 2, \mathrm{Y} 2), \ldots(\mathrm{xm}, \mathrm{Ym})\}$, where $\mathrm{xi} \in \mathrm{X}$ is an instance and $\mathrm{Yi} \subseteq \mathrm{Y}$ is a set of labels $\{$ yi1, yi2 .. yi,li $\}$, yik $\in \mathrm{Y}(\mathrm{k}=1,2 \ldots$ li). Here li denotes the number of labels in Yi.

From Fig. 1 we can see the differences among these learning frameworks. In fact, the multi- learning frameworks are resulted from the ambiguities in representing real-world objects. Multi-instance learning studies the ambiguity in the input space (or instance space), where an object has many alternative input descriptions, i.e., instances; multi-label learning studies the ambiguity in the output space (or label space), where an object has many alternative output descriptions, i.e., labels; while MIML considers the 
ambiguities in both the input and output spaces simultaneously.

In solving real-world problems, having a good representation is often more important than having a strong learning algorithm, because a good representation may capture more meaningful information and make the learning task easier to tackle. Since many real objects are inherited with input ambiguity as well as output ambiguity, MIML is more natural and convenient for tasks involving such objects.

Suppose a multi-label object is described by one instance but associated with $\mathrm{n}$ number of class labels, namely label1, label2 ... labeln. If we represent the multi-label object using a set of $n$ instances, namely instance1, instance $2 . .$. , instance $n$, the underlying information in a single instance may become easier to exploit, and for each label the number of training instances can be significantly increased. So, transforming multi-label examples to MIML examples for learning may be beneficial in some tasks. Moreover, when representing the multi-label object using a set of instances, the relation between the input patterns and the semantic meanings may become more easily discoverable. In some cases, understanding why a particular object has a certain class label is even more important than simply making an accurate prediction, while MIML offers a possibility for this purpose. For example, under the MIML representation, we may discover that one object has label1 because it contains instance $n$; it has label 1 because it contains instance $i$; while the occurrence of both instance 1 and instance $\mathrm{i}$ triggers label $\mathrm{j}$.

\section{MIMLBOOST}

It is used for identifying the equivalence in the traditional supervised learning framework using multi-instance learning as the bridge.

Given any set $\Omega$, let $|\Omega|$ denote its size, i.e., the number of elements in $\Omega$; given any predicate $\Pi$ let [[ $\Pi]$ ] be 1 if $\Pi$ holds and 0 otherwise; given $(\mathrm{Xi}, \mathrm{Yi})$, for any $\mathrm{y} \in \mathrm{Y}$, let $\Psi(\mathrm{Xi}, \mathrm{y})=+1$ if $\mathrm{y} \in \mathrm{Yi}$ and -1 otherwise, where is a function $\Psi: 2^{\mathrm{X}} \times \mathrm{Y} \rightarrow\{-1,+1\}$ which judges whether a label $\mathrm{y}$ is a proper label of $\mathrm{Xi}$ or not. The basic assumption of MimlBoost is that the labels are independent, so that the MIML task can be decomposed into a series of multi-instance learning tasks to solve, by treating each label as a task.

In the first step of MimlBoost, each MIML example $(\mathrm{Xu}$, $\mathrm{Yu})(\mathrm{u}=1,2, \ldots, \mathrm{m})$ is transformed into a set of $|\mathrm{Y}|$ number of multi-instance bags, i.e., $\{[(\mathrm{Xu}, \mathrm{y} 1), \Psi(\mathrm{Xu}, \mathrm{y} 1)],[(\mathrm{Xu}, \mathrm{y} 2)$, $\Psi(\mathrm{Xu}, \mathrm{y} 2)], \ldots,[(\mathrm{Xu}, \mathrm{y}|\mathrm{Y}|), \Psi(\mathrm{Xu}, \mathrm{y}|\mathrm{Y}|)]\}$. Note that $[(\mathrm{Xu}, \mathrm{yv}), \Psi \quad(\mathrm{Xu}, \mathrm{yv})](\mathrm{v}=1,2, \ldots,|\mathrm{Y}|)$ is a labeled multiinstance bag where $(\mathrm{Xu}, \mathrm{yv})$ is a bag containing $\mathrm{n}_{\mathrm{u}}$ number of instances, i.e., $\left\{(\mathrm{xu} 1, \mathrm{yv}),(\mathrm{xu} 2, \mathrm{yv}), \ldots,\left(\mathrm{x}_{\mathrm{u}}, \mathrm{n}_{\mathrm{u}}, \mathrm{yv}\right)\right\}$, and $\Psi$ $(\mathrm{Xu}, \mathrm{yv}) \in\{-1,+1\}$ is the label of this bag.

Thus, the original MIML data set is transformed into a multi-instance data set containing $\mathrm{m} \times|\mathrm{Y}|$ number of bags. Then, from the data set a multi-instance learning function fMIL can be learned, which can accomplish the desired MIML function because

$$
\mathrm{f}_{\mathrm{MIML}}(\mathrm{X} *)=\left\{\mathrm{y} \mid \operatorname{sign}\left[\mathrm{f}_{\mathrm{MIL}}(\mathrm{X} *, \mathrm{y})\right]=+1\right\} .
$$

For convenience, let $(\mathrm{B}, \mathrm{g})$ denote the bag $[(\mathrm{X}, \mathrm{y}),(\mathrm{X}, \mathrm{y})]$, $\mathrm{B} \in \mathrm{B}, \mathrm{g} \in \mathrm{G}$, and $\mathrm{E}$ denotes the expectation. Then, here the goal is to learn a function $\mathrm{F}(\mathrm{B})$ minimizing the bag-level exponential loss

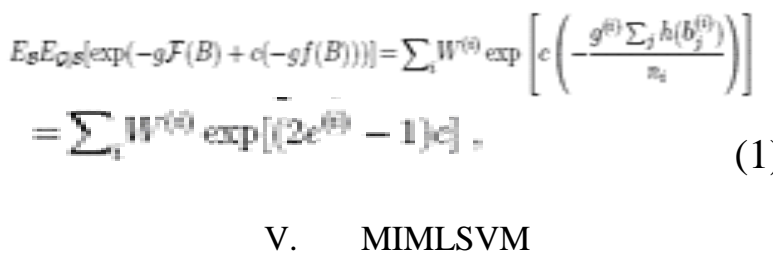

The basic assumption of MimlSvm is that the spatial distribution of the bags carries relevant information, and information helpful for label discrimination can be discovered by measuring the closeness between each bag and the representative bags identified through clustering.

Again, given any set $\Omega$, let $|\Omega|$ denote its size, i.e., the number of elements in $\Omega$; given $(\mathrm{Xi}, \mathrm{Yi})$ and $\mathrm{zi}=\phi(\mathrm{Xi})$ where $\phi: 2 \mathrm{X} \rightarrow \mathrm{Z}$, for any $\mathrm{y} \in \mathrm{Y}$, let $\phi(\mathrm{zi}, \mathrm{y})=+1$ if $\mathrm{y}$ $\in \mathrm{Yi}$ and -1 otherwise, where $\phi$ is a function $\Phi: \mathcal{Z} \times \mathcal{Y} \rightarrow\{-1,+1\}$

In the first step of MimlSvm, the Xu of each MIML example $(\mathrm{Xu}, \mathrm{Yu})(\mathrm{u}=1,2 \ldots \mathrm{m})$ is collected and put into a data set $\Gamma$. Then, in the second step, kmedoids clustering is performed on $\Gamma$. Since each data item in $\Gamma$, i.e. $\mathrm{Xu}$, is an unlabeled multi-instance bag instead of a single instance, Hausdorff distance [26] is employed to measure the distance. The Hausdorff distance is a famous metric for measuring the distance between two bags of points, which has often been used in computer vision tasks; other techniques that can measure the distance between bags of points, such as the set kernel [21], can also be used here. In detail, given two bags A $=\left\{a 1, a 2 \ldots\right.$ an $\left.{ }_{A}\right\}$ and $B=\left\{b 1, b 2 \ldots b_{B}\right\}$, the Hausdorff distance between $\mathrm{A}$ and $\mathrm{B}$ is defined as

$$
d_{B}(A, B)=\max \left\{\max _{a \in A} \min _{b B}\|a-b\|, \max _{b \in B} \min _{a \in A}\|b-a\|\right\},
$$

Where $\|\mathrm{a}-\mathrm{b}\|$ measures the distance between the instances $a$ and $b$, which takes the form of Euclidean distance here.

After the clustering process, the data set $\Gamma$ is divided into $\mathrm{k}$ partitions, whose medoids are $\mathrm{Mt}(\mathrm{t}=1,2, . ., \mathrm{k})$, respectively. With the help of these medoids, the original multi-instance example $\mathrm{Xu}$ is transformed into a k-dimensional numerical vector $\mathrm{zu}$, where the $\mathrm{i}$-th $(\mathrm{i}=1,2 \ldots \mathrm{k})$ component of $\mathrm{zu}$ is the distance between $\mathrm{Xu}$ and $\mathrm{Mi}$, that is, $\mathrm{dH}(\mathrm{Xu}, \mathrm{Mi})$. This process reassembles the constructive clustering process used by Zhou and Zhang [22] in transforming multi-instance examples into single instance examples except that in [22] the clustering is executed at the instance level while here it is executed at the bag level. Thus, the original MIML examples (Xu, Yu) $(u=1$, $2, \ldots \ldots, \mathrm{m})$ have been transformed into multi-label examples $(\mathrm{zu}, \mathrm{Yu})(\mathrm{u}=1,2, \ldots . . \mathrm{m})$, which corresponds to the Step 3 of MimlSvm. 


\section{D-MIMLSVM}

D-MIMLSVM assumes that the labels associated with a bag should have some relatedness; otherwise they should not be associated with the bag simultaneously. To reflect this basic assumption, D-MimlSvm regularizes the empirical loss function in Equation 1 with an additional term $\Omega$ (f):

$$
\Omega(f)+\gamma \cdot V\left(\left\{X_{i}\right\}_{i=1}^{m},\left\{Y_{i}\right\}_{i=1}^{m}, f\right)
$$

Here, $\quad \gamma$ is a regularization parameter balancing the model complexity $\Omega$ (f) and the empirical risk V. Inspired by [23], we assume that the relatedness among the labels can be measured by the mean function w0,

$$
\boldsymbol{w}_{0}=\frac{1}{T} \sum_{t=1}^{T} \boldsymbol{w}_{t}
$$

The original idea in [23] is to minimize $\sum_{t=1}^{T}\left\|w_{t}-w_{0}\right\|^{2}$

regularizer as:

$$
\Omega(f)=\frac{1}{T} \sum_{t=1}^{T}\left\|w_{t}-w_{0}\right\|^{2}+\eta\left\|w_{0}\right\|^{2}
$$

The regularizer can be simplified as:

$$
\Omega(f)=\frac{1}{T} \sum_{t=1}^{T}\left\|w_{t}\right\|^{2}+\mu\left\|w_{0}\right\|^{2}
$$

\section{INSDIF}

For an object associated with multiple class labels, if it is described by only a single instance, the information corresponding to these labels are mixed and thus difficult to learn. The basic assumption of InsDif is that the spatial distribution of instances with different labels encodes information helpful for discriminating these labels, and such information will become more explicit by breaking the single instances into a number of instances each corresponds to one label.

InsDif is a two-stage algorithm, which is based on instance differentiation. In the first stage, InsDif transforms each example into a bag of instances, by deriving one instance for each class label, in order to explicitly express the ambiguity of the example in the input space; in the second stage, an MIML learner is utilized to learn from the transformed data set. For the consistency with our previous description of the algorithm [24], in the current version of InsDif we use a two-level classification strategy, and other MIML algorithms such as DMimlSvm can also be applied.

\section{SUBCOD}

The SubCod (i.e., SUB-COncept Discovery) algorithm which transforms multi-instance single-label examples into MIML examples to exploit the power of MIML. The basic assumption of SubCod is that high-level complicated concepts can be derived by a number of lower-level sub-concepts which are relatively simple and easier for learning, so that we can transform the single-label into a set of labels each corresponds to one sub concept. Therefore, we can learn these labels at first and then derive the high-level complicated label based on them.

A SubCod is a two-stage algorithm. In the first stage, SubCod transforms each single-label example into a multilabel example by discovering and exploiting sub-concepts involved by the original label; this is realized by constructing multiple labels through unsupervised clustering of all instances and then treating each cluster as a set of instances of a separate sub concept. In the second stage, the outputs learned from the transformed data set are used to derive the original labels that are to be predicted; this is realized by using a supervised learning algorithm to predict the original labels from the sub-concepts predicted by an MIML learner.

\section{Performance Evaluation}

We evaluated the performance of the MIML algorithms using average precision, average recall and average F1.

Table1: Over all results of MIML Algorithms

\begin{tabular}{|l|c|c|c|}
\hline \multirow{2}{*}{$\begin{array}{c}\text { Compared } \\
\text { algorithms }\end{array}$} & \multicolumn{3}{|c|}{ Evaluation Criteria } \\
\cline { 2 - 4 } MIML B oost & 0.779 & 0.433 & 0.556 \\
\hline MIML SVM & 0.765 & 0.556 & 0.644 \\
\hline D-MML SVM & 0.783 & 0.587 & 0.671 \\
\hline InSDif & 0.785 & 0.614 & 0.688 \\
\hline SUB COD & 0.712 & 0.530 & 0.532 \\
\hline AdtBoost.MH & 0.718 & $\mathrm{~N} / \mathrm{A}$ & $\mathrm{N} / \mathrm{A}$ \\
\hline RankSvm & 0.746 & 0.529 & 0.620 \\
\hline MlSvm & 0.702 & 0.602 & 0.132 \\
\hline Ml-knn & 0.759 & 0.403 & 0.512 \\
\hline
\end{tabular}

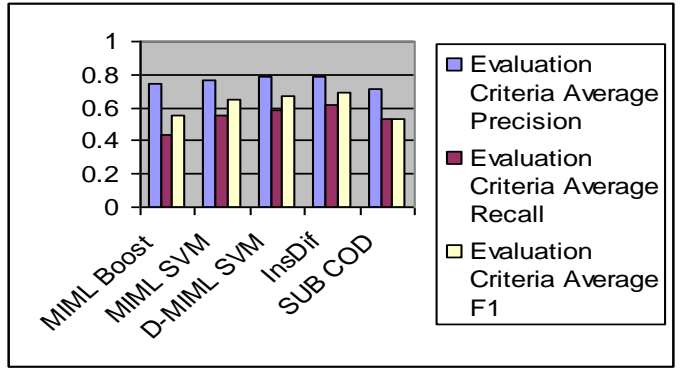

Figure 2: Comparison among MIML Algorithms

As per the results obtained from Table 1 and Figure 2, we can conclude that InsDif performs best in terms of average recall and average F1 and significantly superior with all other multi-label learning algorithms.

\section{CONClusion}

This paper extends our preliminary work to formalize the MIML Multi-Instance Multi-Label learning framework for image annotation, which are associated with multiple instances and multiple class labels. It was inspired by the recognition that when solving real-world problems, having a good representation is more important than having a strong 
learning algorithm because a good representation may capture more meaningful information and make the learning task easier. Since many real objects are inherited with input ambiguity as well as output ambiguity, MIML is more natural and convenient for tasks involving such objects.

To exploit the advantages of the MIML representation, we have reviewed the MIMLBoost algorithm and the MIMLSVM algorithm based on a simple degeneration strategy.

\section{REFERENCES}

[1] Zhi-Hua Zhou, Min-Ling Zhang, Sheng-Jun Huang, Yu-Feng Li "Multi-Instance Multi-Label Learning" In NIPS (2006), pp. 16091616. Key: citeulike: 3398448

[2] Nam Nguyen "A New SVM Approach to Multi-instance Multi-label learning"10.1109/ICDM.2010.109 20 January 2011 IEEE

[3] T. Evgeniou, C. A. Micchelli, and M. Pontil. Learning multiple tasks with kernel methods. Journal of Machine Learning Research, 6:615637, 2005.

[4] R. E. Schapire and Y. Singer. BoosTexter: A boosting-based system for text categorization. Machine Learning, 39(2-3):135-168, 2000.

[5] F. De Comit'e, R. Gilleron, and M. Tommasi. Learning multi-label alternating decision tree from texts and data. In Proceedings of the 3rd International Conference on Machine Learning and Data Mining in Pattern Recognition, pages 35-49, Leipzig, Germany, 2003.

[6] Y. Freund and L. Mason. The alternating decision tree learning algorithm. In Proceedings of the 16th International Conference on Machine Learning, pages 124-133, Bled, Slovenia, 1999.

[7] McCallum. Multi-label text classification with a mixture model trained by EM. In Working Notes of the AAAI'99 Workshop on Text Learning, Orlando, FL, 1999.

[8] N. Ueda and K. Saito. Parametric mixture models for multi-labeled text. Advances in Neural Information Processing Systems 15, pages 721-728. MIT Press, Cambridge, MA, 2003.

[9] Y. Chen, J. Bi, and J. Z. Wang. MILES: Multiple-instance learning via embedded instance selection. IEEE Transactions on Pattern Analysis and Machine Intelligence, 28(12):1931-1947, 2006.

[10] Y. Chen and J. Z. Wang. Image categorization by learning and reasoning with regions. Journal of Machine Learning Research, 5:913939, 2004.

[11] C. Yang and T. Lozano-P'erez. Image database retrieval with multipleinstance learning techniques. In Proceedings of the 16th International Conference on Data Engineering, pages 233-243, San Diego, CA, 2000.

[12] Q. Zhang, W. Yu, S. A. Goldman, and J. E. Fritts. Content-based image retrieval using multiple-instance learning. In Proceedings of the 19th International Conference on Machine Learning, pages 682-689, Sydney, Australia, 2002.

[13] S. Andrews, I. Tsochantaridis, and T. Hofmann. Support vector machines for multiple-instance learning. Advances in Neural Information Processing Systems 15, pages 561-568. MIT Press, Cambridge, MA, 2003.

[14] B. Settles, M. Craven, and S. Ray. Multiple-instance active learning. In J. C.Platt, D. Koller, Y. Singer, and S. Roweis, editors, Advances in Neural Information Processing Systems 20, pages 1289-1296. MIT Press, Cambridge, MA, 2008.

[15] Z.-H. Zhou, K. Jiang, and M. Li. Multi-instance learning based web mining. Applied Intelligence, 22(2):135-147, 2005.

[16] Z. Jorgensen, Y. Zhou, and M. Inge. A multiple instance learning strategy for combating good word attacks on spam filters. Journal of Machine Learning Research, 8:993-1019, 2008.

[17] G. Ruffo. Learning single and multiple instance decision trees for computer security applications. $\mathrm{PhD}$ thesis, Department of Computer Science, University of Turin, Torino, Italy, 2000.

[18] P. Viola, J. Platt, and C. Zhang. Multiple instances boosting for object detection. In Y. Weiss, B. Sch"olkopf, and J. Platt, editors, Advances in Neural Information Processing Systems 18, pages 1419-1426. MIT Press, Cambridge, MA, 2006.

[19] C. Zhang and P. Viola. Multiple-instance pruning for learning efficient cascade detectors. In J. C. Platt, D. Koller, Y. Singer, and S. Roweis, editors, Advances in Neural Information Processing Systems 20, pages 1681-1688. MIT Press, Cambridge, MA, 2008.
[20] G. Fung, M. Dundar, B. Krishnappuram, and R. B. Rao. Multiple instance learning for computer aided diagnosis. In B. Sch"olkopf, J. Platt, and T. Hofmann, editors, Advances in Neural Information Processing Systems 19, pages 425-432. MIT Press, Cambridge, MA, 2007.

[21] T. G̈artner, P. A. Flach, A. Kowalczyk, and A. J. Smola. Multiinstance kernels. In Proceedings of the 19th International Conference on Machine Learning, pages179-186, Sydney, Australia, 2002.

[22] Z.-H. Zhou and M.-L. Zhang. Solving multi-instance problems with classifier ensemble based on constructive clustering. Knowledge and Information Systems, 11(2):155-170, 2007.

[23] T. Evgeniou, C. A. Micchelli, and M. Pontil. Learning multiple tasks with kernel methods. Journal of Machine Learning Research, 6:615637, 2005.

[24] M.-L. Zhang and Z.-H. Zhou. Multi-label learning by instance differentiation. In Proceedings of the 22nd AAAI Conference on Artificial Intelligence, pages 669-674, Vancouver, Canada, 2007.

\section{Authors PROFILE}

C. Lakshmi Devasena is presently doing $\mathrm{PhD}$ in Karpagam University, Coimbatore, Tamilnadu, India. She has completed M.Phil. (computer science) in the year 2008 from Bharathidasan University and MCA degree in 1997 from RVS College of Engineering and Technology, Dindigul (Madurai Kamaraj University) and B.Sc (Mathematics) in 1994 from Gandhigram Rural Institute. She has Eight years of teaching experience and Two years of industrial experience. Her area of research interest is Image processing and Data mining. She has published 10 papers in International Journals and Presented 18 papers in National and international conferences. At Present, she is working as Lecturer in Karpagam University, Coimbatore.

M. Hemalatha completed MCA MPhil., PhD in Computer Science and currently working as an Assistant Professor and Head, Department of software systems in Karpagam University. Ten years of Experience in teaching and published more than fifty papers in International Journals and also presented seventy papers in various National conferences and one international conferences Area of research is Data mining, Software Engineering, bioinformatics, Neural Network. She is also reviewer in several National and International journals.

T. Sumathi is presently doing $\mathrm{PhD}$ in Karpagam University, Coimbatore, Tamilnadu, India and has completed M.Phil (computer science) in the year 2006 and MCA degree in 1999 and B. Sc(computer science) in 1996. Major research area is Image processing and title for the research is image annotation. At Present, she is working as Lecturer in Karpagam University, Coimbatore. 